\title{
Comparative Transcriptomes Profiling of Photoperiod-sensitive Male Sterile Rice Nongken 58S During the Male Sterility Transition between Short-day and Long-day
}

Wei Wang, Zhenwei Liu, Zhibin Guo, Gaoyuan Song, Qin Cheng, Daiming Jiang, Yingguo Zhu and Daichang Yang*

\begin{abstract}
Background: Photoperiod-sensitive genic male sterile (PGMS) rice, Nongken 58S, was discovered in 1973. It has been widely used for the production of hybrid rice, and great achievements have been made in improving rice yields. However, the mechanism of the male sterility transition in PGMS rice remains to be determined.

Results: To investigate the transcriptome during the male sterility transition in PGMS rice, the transcriptome of Nongken 585 under short-day (SD) and long-day (LD) at the glume primordium differentiation and pistil/stamen primordium forming stages was compared. Seventy-three and 128 differentially expressed genes (DEGs) were identified at the glume primordium differentiation and pistil/stamen primordium forming stages, respectively. Five and 22 genes were markedly up-regulated ( $\geq 5$-fold), and two and five genes were considerably down-regulated ( $\geq 5$-fold) under SD during the male sterility transition. Gene ontology annotation and pathway analysis revealed that four biological processes and the circadian rhythms and the flowering pathways coordinately regulated the male sterility transition. Further quantitative PCR analysis demonstrated that the circadian rhythms of OsPRR1, OsPRR37, OsGl, Hd1, OsLHY and OsDof in leaves were obviously different between Nongken $58 \mathrm{~S}$ and Nongken 58 under LD conditions. Moreover, both OsPRR37 and Hd1 in the inflorescence displayed differences between Nongken $58 \mathrm{~S}$ and Nongken 58 under both LD and SD conditions.
\end{abstract}

Conclusion: The results presented here indicate that the transcriptome in Nongken 585 was significantly suppressed under LD conditions. Among these DEGs, the circadian rhythm and the flowering pathway were involved in the male sterility transition. Furthermore, these pathways were coordinately involved in the male sterility transition in PGMS rice.

Keywords: transcriptome profiling, PGMS, male sterility transition, circadian rhythm, flowering

\section{Background}

Photoperiod-sensitive genic male sterile (PGMS) rice, Nongken 58S, was discovered as a spontaneous mutant in the japonica rice cultivar Nongken 58 (Oryza sativa ssp. japonica) grown in Hubei Province, China in 1973 [1]. It has since been used for production of hybrid rice, and during the past two decades, great achievements have been made in improving rice yields in China using

\footnotetext{
* Correspondence: dyang@whu.edu.cn

State Key Laboratory of Hybrid Rice, College of Life Sciences, Wuhan University, North Road of Luoshi, Wuhan, Hubei Province, China
}

two-line hybrid rice. Several important features of Nongken $58 \mathrm{~S}$ have been characterised. Its fertility is highly regulated by day length at specific inflorescence developmental stages. Complete male sterility can be induced when the day length is greater than $14 \mathrm{~h}$ from the glume primordium differentiation stage to the pollen mother cell forming stage. However, male fertility returns gradually when the day length is shorter than 14 $\mathrm{h}$ [1]. This is called the second photoperiod phenomenon [2], referring to the short day photoperiod that rice requires for the transition from vegetative growth
C Biomed Central

(c) 2011 Wang et al; licensee BioMed Central Ltd. This is an Open Access article distributed under the terms of the Creative Commons Attribution License (http://creativecommons.org/licenses/by/2.0), which permits unrestricted use, distribution, and reproduction in any medium, provided the original work is properly cited. 
to reproductive growth [2]. This agronomic trait is genetically controlled by a recessive locus within the nuclear genome. However, the genetic loci vary depending on the genetic background of the recipient parents. That is, when crossed with a less photoperiod-sensitive cultivar, the genes for the trait appear as two or three loci [3-7]. The photoperiod sensitivity can also be affected by temperature when the pms gene is crossed into a temperature-sensitive cultivar. Previous studies have indicated that phytochromes and cryptochromes are involved in the male sterility transition [2]. Because male sterility is highly and coordinately regulated by day length and temperature, this leads to difficulty in the accurate identification of the male sterile phenotype in the segregation populations between japonica and indica under natural conditions. Although it would be of great value to breeders, this makes mapping the pms gene challenging $[3-5,8,9]$. It is important to understand the molecular mechanism of the male sterility transition and to explore whether the light or circadian rhythm signal transduction pathway is involved in this process. It may also be useful to understand how light or the circadian rhythm regulates microsporocyte development. This information could assist breeders in selecting recipient parents for molecular breeding programs.

Circadian rhythms, in general, take the form of sinusoidal waves that can be described using mathematical terms such as period, phase and amplitude [10-12]. The expression of several genes has been found to be associated with the circadian rhythm in Arabidopsis. These include TIMING OF CAB EXPRESSION1 (TOC1) [13], LATE ELONGATED HYPOCOTYL (LHY) [14], CIRCADIAN AND CLOCK ASSOCIATED1 (CCA1) [15] and GIGANTEA (GI). TOC1, LHY, CCA1 and unknown factor Y $[16,17]$ comprise interlocked transcriptional feedback loops. These feedback loops play important roles in the plant central clock. These loops integrate environmental factors, such as light and temperature, into the central clock through the input signaling pathway and import the rhythm signal into downstream signaling pathways through output signaling pathways.

Two main pathways controlling flowering time are found in rice. One is the EARLY HEADING DATE1 (Ehd1)/HEADING DATE3a (Hd3a)/RICE FLOWERING LOCUS T1 (RFT1)-dependent pathway [18-22]. In this pathway, the upstream gene Ehd2/RID1/OsId1, under short-day (SD) conditions, activates Ehd1. Ehd1 then activates $H d 3 a / R F T 1$ to promote rice flowering. By contrast, Ehd 1 is suppressed under long-day (LD) conditions. Hd3a/RFT1 is also suppressed, leading to the inhibition of rice flowering [21]. The other pathway is the HEADING DATE1 (Hd1)-Hd3a/RFT1-dependent pathway $[18,23]$. In this pathway, $H d 1$ is activated by OsGI under LD or SD conditions. Hd1 then activates
Hd3a/RFT1 expression and promotes flowering under SD conditions. However, Hd1 suppresses Hd3a/RFT1 expression and inhibits flowering under LD conditions $[18,24,25]$. Previous research has indicated that circadian rhythms and day length are not only involved in the promotion of reproductive organs from the vegetative stage in higher plants $[11,26,27]$, but are also involved in various physiological processes, including photosynthesis [28,29], starch metabolism [30-32], phytohormone response [32-35], hypocotyl elongation [36,37], and plant-pathogen interaction [38]. However, the involvement of these two signaling pathways in the male sterility transition has not been reported.

This paper reports the comparison of the transcriptomes of Nongken 58S under SD and LD conditions during the male sterility transition. The repressive expression profile under LD conditions identified 183 differentially expressed genes (DEGs). Gene ontology (GO) and pathway analysis of the DEGs revealed that the circadian rhythm and the flowering pathways were involved in the male sterility transition. In further analysis, qPCR results indicated that the circadian rhythms of OsPRR1, OsPRR37, OsGI, Hd1, OsLHY and OsDof in Nongken $58 \mathrm{~S}$ were significantly different from those in Nongken 58. This suggests that the circadian rhythm and the flowering pathways were coordinately involved in regulation of the male sterility transition.

\section{Methods}

\section{Plant materials}

The varieties Nongken 58 and the mutant Nongken $58 \mathrm{~S}$ (Oryza sativa L. ssp. japonica) were used for this study. All plants were grown under natural conditions at Wuhan University Campus. SD (10-h light/14-h dark) treatment was conducted when the seedlings had greater than five leaves. Differentiation from vegetative growth to reproductive growth was promoted after 10-12 days of SD treatment. When the inflorescences had developed to the secondary branch differentiation stage, the rice plants were divided into two groups for LD (15-h light/9-h dark) and SD treatment, respectively.

\section{RNA isolation and CDNA synthesis}

For total RNA isolation, the second fully expanded leaf was harvested at two developmental stages: glume primordium differentiation and pistil/stamen primordium formation. For diurnal expression profile analysis, the leaves were harvested at $4 \mathrm{~h}$ intervals over 1 day, frozen immediately in liquid nitrogen, and stored at $-80^{\circ} \mathrm{C}$ until use [39]. The samples are annotated in Additional file 1, Table S1.

A TRIzol Reagent Kit (Invitrogen, Carslbad, USA) was used for total RNA isolation following the manufacturer's instructions. Total RNA was treated with RNase- 
free DNase I (New England Biolabs, Hitchin, UK) to remove DNA contamination before cDNA synthesis. Two micrograms of total RNA and Oligo (dT) were used as template and primers for first strand cDNA synthesis by M-MLV reverse transcriptase (Promega, Madison, USA).

\section{Microarray analysis procedure and comparison strategy}

Two micrograms of total RNA were used for doublestranded cDNA synthesis, and biotin-tagged cRNA was prepared using a MessageAmp ${ }^{\mathrm{TM}}$ II cRNA Amplification Kit according to the manufacturer's instructions. The resulting bio-tagged cRNA was fragmented into strands of 35-200 bases according to Affymetrix protocols. The fragmented cRNA was hybridised to an Affymetrix GeneChip Rice Genome Array containing 48,564 and 1,260 transcripts representing the japonica and indica cultivars, respectively. The microarray and data analysis were contracted to CapitalBio Corporation (Beijing, China). The hybridisation was performed at $45^{\circ} \mathrm{C}$ with rotation for $16 \mathrm{~h}$ (GeneChip Hybridization Oven 640, Affymetrix). The GeneChip arrays were washed and then stained (streptavidin-phycoerythrin) on an Affymetrix Fluidics Station 450. Scanning was conducted using a GeneChip Scanner 3000. All chip data represented biological triplicates.

The scanned images were examined by visual inspection and then processed to generate raw data using the default setting of GeneChip Operating Software (GCOS 1.4). dChip software was used to perform invariant-set normalization to normalize the arrays according to dChip users' manual. 5\% of Perfect-Match (PM) probe signals were used as background removal. Annotation of the samples is shown in Additional file 1, Table S1. The samples were compared as follows: G-SSD2 vs. G-SLD2 and P-SSD2 vs. P-SLD2. The criteria for determining up- and down-regulated genes were fold changes (FCs) of $\geq 2$ and $\leq 0.5$, respectively. The complete microarray data have been deposited in NCBI's Gene Expression Omnibus and are accessible through GEO series accession number GSE29820.

\section{Cluster, gene ontology annotation and pathway analysis} All microarray data were analysed using Significant Analysis of Microarray (SAM) 3.02 software. Genes with FC $\geq 2$ or $\mathrm{FC} \leq 0.5$ were chosen for the $\mathrm{t}$-test, and genes with $P$ values $<0.05$ were chosen for further analysis. Cluster analysis was performed using Cluster 3.0 software. GO annotation was performed using the GeneOntology Enrichment Analysis Software Toolkit (http:// omicslab.genetics.ac.cn/GOEAST/php/affymetrix.php) $[40,41]$. GOs with $P$ values $<0.01$ were selected. Pathway analysis was accomplished using the MAS 3.0 system (http://www.capitalbio.com/index.asp) (CapitalBio
Corporation, Beijing, China) and by searching the Kyoto Encyclopedia of Genes and Genomes, BioCarta and GenMAPP databases. Pathways with $P$ values $<0.01$ were selected.

\section{Quantitative PCR analysis}

For quantitative PCR, the cycling conditions were $95^{\circ} \mathrm{C}$ for $10 \mathrm{~min}$, followed by 40 cycles of $95^{\circ} \mathrm{C}$ for $10 \mathrm{~s}$ and $60^{\circ} \mathrm{C}$ for $30 \mathrm{~s}$, and then a melting curve from $60^{\circ} \mathrm{C}$ to $95^{\circ} \mathrm{C}$ in $0.3^{\circ} \mathrm{C}$ increments. Negative controls without the template DNA were used to ensure that primer dimers were not interfering with amplification. qPCR data was captured and analysed using StepOne software (ver. 2.0). The relative gene expression levels in each cDNA sample were obtained by normalisation to ACTIN1 using the formula $2^{-(\mathrm{CT} \text { gene - CT actin1) }}$ [42]. The primers used in this study are listed in Additional file 2, Table S2. All qPCR data is the result of biological triplicates.

\section{Results}

\section{Dynamic transcriptome profiling of photoperiod} treatment during the male sterility transition

As shown in Figure 1, pollen abortion was induced in Nongken 58S under LD conditions (15-h light/9-h dark) from the glume primordium differentiation to the pollen mother cell forming stages (Figure $1 \mathrm{~A}-\mathrm{C}$ ) $[2,43]$. Under SD conditions (10-h light/14-h dark), the pollen partially regained fertility (Figure 1E, G). By contrast, pollen fertility in Nongken 58 was not affected (Figure 1D, F). These results indicated that the fertility of PGMS could not be completely restored using normal photoperiod conditions, implying that some defective signaling pathway may be constitutively affected. To investigate the transcriptome changes during the male sterility transition process in PGMS rice, we chose the leaves, light signal receiver, as experimental material at photoperiodsensitive stage. Based on preliminary experiments, the diurnal expression profiles of those genes peaked at 02:00 h (data not shown). Thus, 02:00 h was selected as the expression profile time point for the microarray. Affymetrix GeneChips were used for global transcriptome profiling analysis. The correlation coefficients between different biological repeats were calculated, and the result indicates that the profilings were reproducible (Additional file 3, Table S3). A total of 20,444 and 19,964 transcripts were detected $P$ values $<0.05$ in the comparison of G-SSD2 vs. G-SLD2 and P-SSD2 vs. PSLD2, respectively. In comparing the SD and LD conditions at the two developmental stages, 183 DEGs with two fold changes were detected $P<0.05$.

The DEGs were categorised using Cluster 3.0 software (Figure 2A, B, C). The number of DEGs with FC $\geq 2$ or $\leq 0.5$ increased significantly as inflorescence development proceeded (Figure 2D, E; Additional file 4, Table 


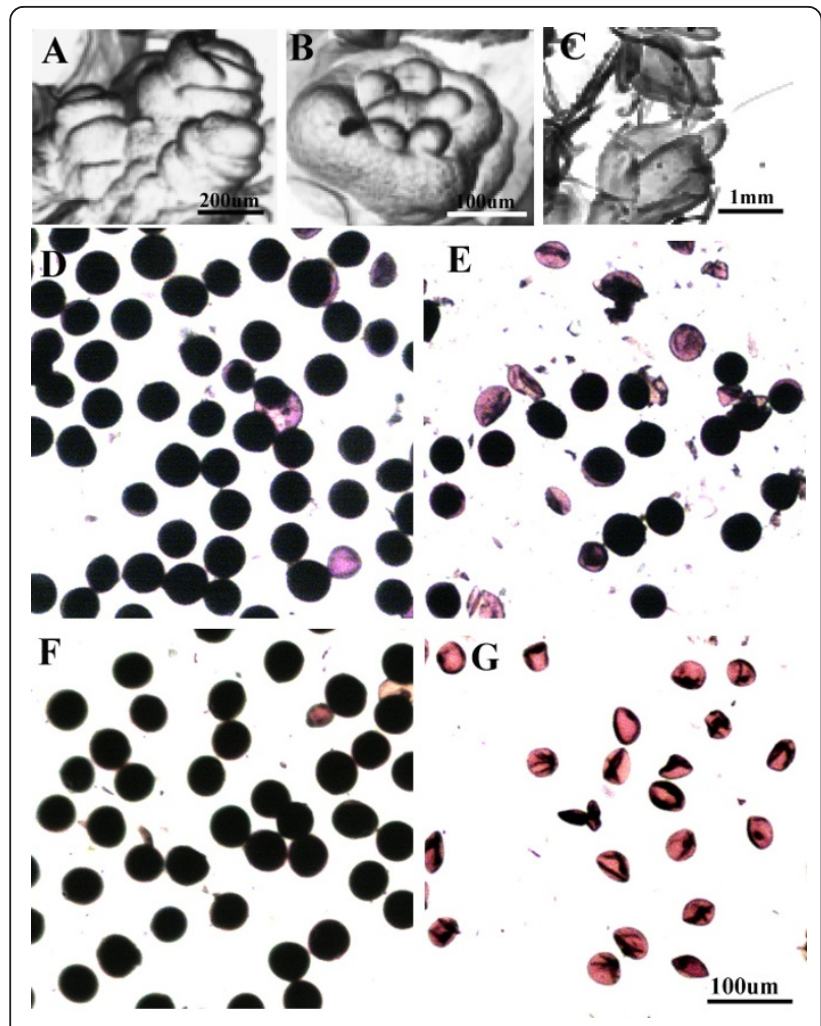

Figure 1 Pollen fertility of Nongken $58 \mathrm{~S}$ and Nongken 58 under LD and SD conditions Scanning microscope images of rice inflorescence developmental stages and the pollen fertility of Nongken 58 and Nongken 585 under LD and SD conditions. (A) glume primordium differentiation stage, Bar $=200 \mu \mathrm{m}$; (B) pistil/ stamen primordium forming stage, $B a r=100 \mu \mathrm{m}$; (C) pollen mother cell forming stage, Bar $=1 \mathrm{~mm}$; Pollen fertility of Nongken 58 (D) and Nongken $58 \mathrm{~S}$ (E) under SD conditions. Pollen fertility of Nongken $58(\mathrm{~F})$ and Nongken $58 \mathrm{~S}(\mathrm{G})$ under LD conditions Bar = $100 \mu \mathrm{m} ; \mathrm{D}, \mathrm{E}, \mathrm{F}$ have the same magnification as G. Pollen was stained with $1 \% \mathrm{I}_{2} / \mathrm{Kl}$ and observed under a light microscope.

S4; Additional file 5, Table S5). At the glume primordium differentiation and the pistil/stamen primordium forming stages, 73 and 128 DEGs with FC $\geq 2$ or $\leq 0.5$ $(P<0.05)$ were detected, respectively (Figure 2D). Five genes were identified as markedly up-regulated $(\geq 5$ fold) and two genes were noted as considerably downregulated ( $\geq 5$-fold) under SD conditions at the glume primordium stage (Figure 2E; Additional file 4, Table S4). Twenty-two genes were considerably up-regulated ( $\geq 5$-fold), and only five genes were down-regulated ( $\geq$ 5 -fold) at the pistil/stamen primordium forming stage (Figure 2E, Additional file 5, Table S5). These results indicated that more genes were suppressed than were activated under LD conditions. This suggests that transcriptome profiling in Nongken $58 \mathrm{~S}$ was significantly suppressed, and the repression effects were markedly intensified as inflorescence development proceeded. This is consistent with previous studies in which pollen fertility was found to decrease as LD treatment increased [44].

Defining DEGs for GO annotation and pathway analysis To annotate the DEGs, the sequences were uploaded to the GOEAST website for GO annotation analysis $[40,41,45]$. The results indicated that some biological processes were significantly affected during the second photoperiod under LD conditions. Four GO biological processes related to the regulation of the transition from the vegetative to the reproductive phase (GO:0048510), the regulation of SD photoperiodism (GO:0048572), the photoperiodism (GO:0009648), and inflorescence development (GO:0010229) were investigated during the second photoperiod (Table 1). The results implied that these processes were related to the male sterility transition in PGMS rice.

To define the potential signal transduction pathways, the DEGs were uploaded to the MAS 3.0 system. Five pathways and seven pathways were identified from the transcriptome data for G-SSD2 vs. G-SLD2 and P-SSD2 vs. P-SLD2, respectively (Table 2 ). The circadian rhythm pathway was top ranked $(P=6.57 \mathrm{E}-06$ and $1.38 \mathrm{E}-05)$ in both inflorescence developmental stages (Table 2). The results indicated that three genes, OsPRR1 (homologous to PRR1/TOC1 in Arabidopsis), OsLHY (homologous to $L H Y$ in Arabidopsis) and MYB transcription factor (LOC_Os06g51260), belong to the circadian rhythm pathway. This suggests that the circadian rhythm may be a principal signal transduction pathway in the male sterility transition in PGMS.

\section{Circadian rhythm involvement in the male sterility transition}

As mentioned previously, several clock genes [OsPRR1 (LOC_Os02g40510), OsPRR37 (LOC_Os07g49460), OsGI (LOC_Os01g08700), OsLHY (LOC_Os08g06110) and $M Y B$ transcription factor (LOC_Os06g51260)] were identified (Additional file 4, Table S4, Additional file 5, Table S5). Among these genes, OsPRR37 and OsGI were upregulated 3.0-fold and 8.3-fold, respectively; OsLHY and the $M Y B$ transcription factor were down-regulated 2.4fold and 2.6-fold, respectively. No difference in OsPRR1 expression was detected in the G-SSD2 vs. G-SLD2 data (Additional file 4, Table S4); in the P-SSD2 vs. P-SLD2 data, OsPRR1 was up-regulated 2.0-fold and the $M Y B$ transcription factor was down-regulated 4.4-fold. No differences in OsPRR37, OsGI and OsLHY were detected at this inflorescence developmental stage. The fold changes indicate that these clock genes were activated or repressed under LD conditions in Nongken 58S, implying that they may participate in the male sterility transition.

Because gene transcription is dynamic, the microarray data reflect expression differences at 02:00 h only. 


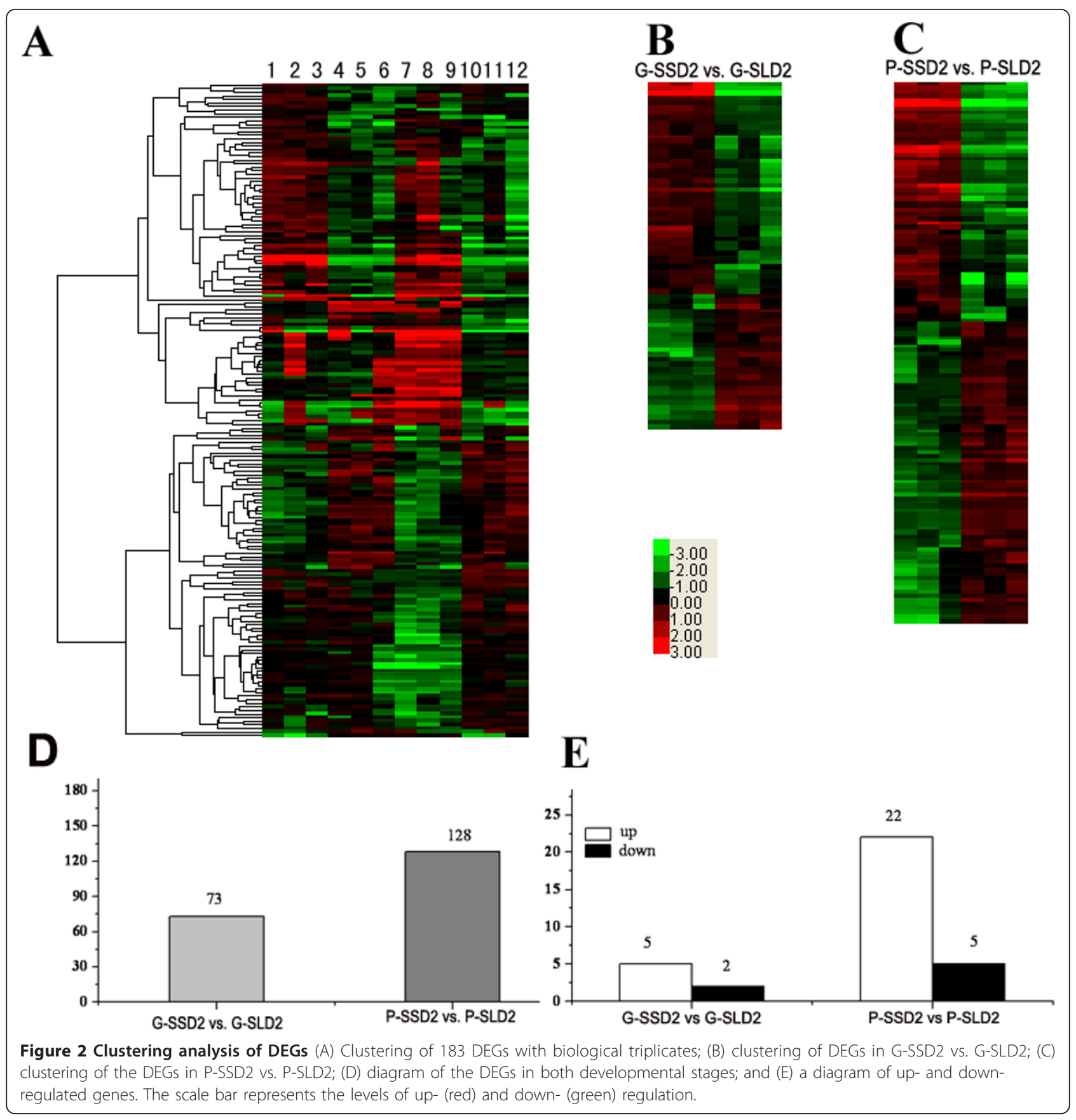

Therefore, the diurnal expression profiles of these genes were analysed in both Nongken $58 \mathrm{~S}$ and Nongken 58 at the glume primordium differentiation and pistil/stamen primordium forming stages under LD and SD conditions. OsPRR1, OsPRR37 and OsGI exhibited the similar circadian rhythm patterns under LD conditions (Figure $3 \mathrm{~A}-\mathrm{C})$. For three of these genes, the differences between Nongken $58 \mathrm{~S}$ and Nongken 58 only occurred at 18:00 h at the glume primordium differentiation stage. The expression levels in Nongken $58 \mathrm{~S}$ were significantly higher than in Nongken 58. No difference was found at the pistil/stamen primordium forming stage under LD and SD conditions (Figure 3A-C, Additional file 6, Figure S1A-C). These results indicate a circadian rhythm difference in the expression of the three genes between Nongken $58 \mathrm{~S}$ and Nongken 58 [12].

$O s L H Y$, a dawn-phased gene, is repressed during the day and activated at night. Its expression level differed significantly between Nongken $58 \mathrm{~S}$ and Nongken 58 at 22:00 h, 02:00 h, 06:00 $\mathrm{h}$ at the glume primordium 
Table 1 GO biological process of DEGs ${ }^{1}$

\begin{tabular}{|c|c|c|c|}
\hline GO term & Gene number. & $P$ value ${ }^{2}$ & $Q$ value \\
\hline \multicolumn{4}{|l|}{ G-SSD2 vs. G-SLD2 } \\
\hline GO:0006350 transcription & 8 & 0.0000034 & 0.0000562 \\
\hline GO:0048510 regulation of timing of transition from vegetative to reproductive phase & 1 & 0.000759 & 0.004176 \\
\hline GO:0048572 short-day photoperiodism & 1 & 0.000759 & 0.004176 \\
\hline GO:0009648 photoperiodism & 1 & 0.001518 & 0.005566 \\
\hline GO:0010229 inflorescence development & 1 & 0.002276 & 0.007426 \\
\hline GO:0006950 response to stress & 6 & 0.005823 & 0.012811 \\
\hline GO:0009628 response to abiotic stimulus & 5 & 0.006268 & 0.012837 \\
\hline GO:0006352 transcription initiation & 1 & 0.007944 & 0.014171 \\
\hline GO:0006265 DNA topological change & 1 & 0.007944 & 0.014171 \\
\hline \multicolumn{4}{|l|}{ P-SSD2 vs. P-SLD2 } \\
\hline GO:0048510 regulation of transition from vegetative to reproductive phase & 1 & 0.001156 & 0.021567 \\
\hline GO:0048572 short-day photoperiodism & 1 & 0.001156 & 0.021567 \\
\hline GO:0009648 photoperiodism & 1 & 0.002311 & 0.021567 \\
\hline GO:0010229 inflorescence development & 1 & 0.003464 & 0.02425 \\
\hline
\end{tabular}

${ }^{1}$ DEGs with $\mathrm{FC} \geq 2$ or $\leq 0.5$ and $P<0.05$ were chosen for GO annotation.

${ }^{2} \mathrm{GO}$ terms with $P<0.01$ are listed in the table.

differentiation stage, and 06:00 $\mathrm{h}$ at the pistil/stamen primordium forming stage (Figure $3 \mathrm{E}$ ). However, no difference was detected under SD conditions (Additional file 6, Figure S1E). The results also indicated circadian rhythm differences between Nongken $58 \mathrm{~S}$ and Nongken 58. OsDof (LOC_Os01g15900), a Dof-type zinc finger (Additional file 4, Table S4), showed a similar diurnal pattern to OsLHY. The major differences between Nongken $58 \mathrm{~S}$ and Nongken 58 occurred at 22:00 h and 02:00 $\mathrm{h}$ at the glume primordium differentiation stage, and at 22:00 h, 02:00 h, and 06:00 h at the pistil/stamen primordium forming stage (Figure 3F).

Table 2 Signal transduction pathway analysis of DEGs ${ }^{1}$

\begin{tabular}{|c|c|c|c|}
\hline KEGG pathway & $\begin{array}{c}\text { Gene } \\
\text { number }\end{array}$ & $P$ value $^{2}$ & $Q$ value \\
\hline \multicolumn{4}{|c|}{ G-SSD2 vs. G-SLD2 } \\
\hline Circadian rhythm & 2 & 0.00000657 & 0.0000131 \\
\hline Thiamine metabolism & 1 & 0.000573 & 0.000573 \\
\hline Streptomycin biosynthesis & 1 & 0.001065 & 0.00071 \\
\hline Inositol phosphate metabolism & 1 & 0.006783 & 0.003235 \\
\hline Starch and sucrose metabolism & 1 & 0.008086 & 0.003235 \\
\hline \multicolumn{4}{|c|}{ P-SSD2 vs. P-SLD2 } \\
\hline Circadian rhythm & 2 & 0.0000138 & 0.0000276 \\
\hline Thiamine metabolism & 1 & 0.000803 & 0000803 \\
\hline Cyanoamino acid metabolism & 1 & 0.002521 & 0.001318 \\
\hline Ubiquinone biosynthesis & 1 & 0.002636 & 0.001318 \\
\hline Nitrogen metabolism & 1 & 0.004123 & 0.001412 \\
\hline Galactose metabolism & 1 & 0.004237 & 0.001412 \\
\hline $\begin{array}{c}\text { Alanine and aspartate } \\
\text { metabolism }\end{array}$ & 1 & 0.005494 & 0.00157 \\
\hline
\end{tabular}

${ }^{1}$ DEGs with FC $\geq 2$ or $\leq 0.5$ and $P<0.05$ were chosen for pathway analysis.

${ }^{2}$ Pathways with $P<0.01$ are listed in the table.
Taken together, these differences only occurred under LD conditions at the photoperiod-sensitive developmental stage. Based on the genetic background of Nongken $58 \mathrm{~S}$ and Nongken 58, these genes were all related to the male sterility transition and the rice circadian rhythm was involved in this process.

\section{Flowering gene $H d 1$ is involved in the male sterility transition under LD conditions}

Ehd1, Hd3a, RFT1 and OsMADS1 were all significantly suppressed under LD conditions in Nongken 58S (Additional file 4, Table S4; Additional file 5, Table S5). Ehd1, $H d 3 a, R F T 1$ are crucial genes for rice flowering [18-21,23], and OsMADS1 plays key roles in specifying floral organ and meristem identity in rice [46-48]. Ehd2 and $H d 1$ are also important components of the flowering pathway. To determine whether these genes were involved in the male sterility transition, the diurnal expression profiles of these genes were analysed. $H d 1$ was the only gene exhibiting different daily expression profiles between Nongken 58S and Nongken 58 under LD conditions (Figure 3D and Additional file 7, Figure S2). The main differences occurred at the pistil/stamen primordium forming stage. The transcription levels at 22:00 $\mathrm{h}$ and 06:00 $\mathrm{h}$ in Nongken $58 \mathrm{~S}$ were significantly different from those in Nongken 58, indicating that the peak phase of $H d 1$ was at 06:00 h in Nongken 58 and it was shifted forward to $22: 00 \mathrm{~h}$ in Nongken $58 \mathrm{~S}$. The phase is a mathematical term of plant circadian rhythms [12]. The phase-shift indicated that the circadian rhythm of $\mathrm{Hd} 1$ in Nongken $58 \mathrm{~S}$ was notably different from that in Nongken 58, while the flowering time of Nongken 58 is similar to that of Nongken $58 \mathrm{~S}$ under LD conditions. 


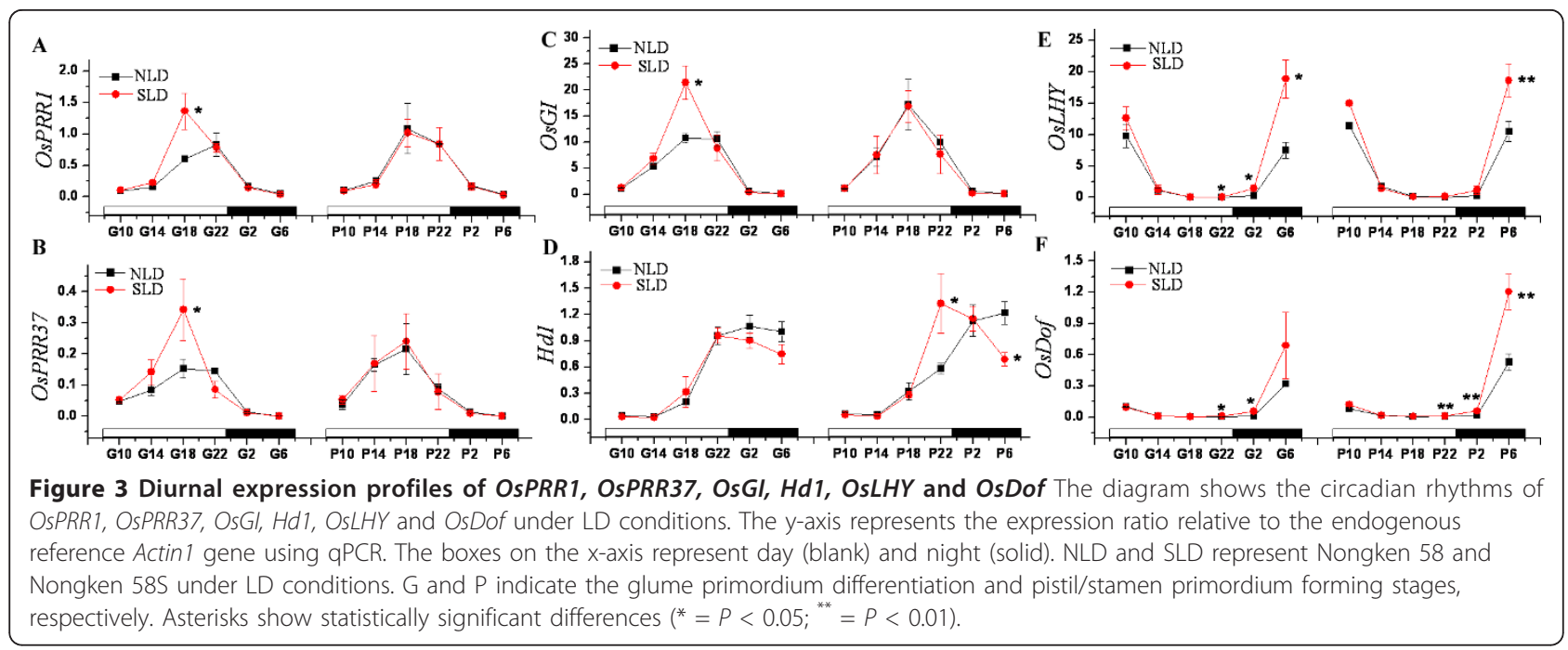

This suggests that $H d 1$ plays a role in the male sterility transition in PGMS.

OsPRR37 and $H d 1$ were differentially expressed in the inflorescences of Nongken $58 \mathrm{~S}$ and Nongken 58

Because of light signals received from the leaf, knowledge of the expression profiles of DEGs in the inflorescence is essential. Further qPCR of the expression patterns of these genes in the inflorescence showed that OsPRR1, OsGI, OsLHY, OsDof expression was similar in Nongken 58S and Nongken 58 under LD and SD conditions (Additional file 8, Figure S3). Only OsPRR37 and $H d 1$ were differentially expressed in the inflorescences of Nongken $58 \mathrm{~S}$ and Nongken 58 at the pistil/stamen primordium forming stage (Figure 4). The expression level of OSPRR37 in Nongken 58S was significantly lower than that in Nongken 58 at 17:00 h under LD conditions (Figure 4A), and the level of OsPRR37 was significantly lower than that in Nongken 58 at 20:00 h under SD conditions (Figure 4B). The expression level of $\mathrm{Hd} 1$ in Nongken $58 \mathrm{~S}$ was significantly greater than that in Nongken 58 at 20:00 h and 02:00 h under LD conditions (Figure 4C), but was significantly lower than that in Nongken at 20:00 h. However, the transcription level in Nongken 58S at 02:00 h was higher than that in Nongken 58. These results suggest that OsPRR37 and $H d 1$ are likely involved in the male sterility transition directly under LD conditions in the inflorescence.

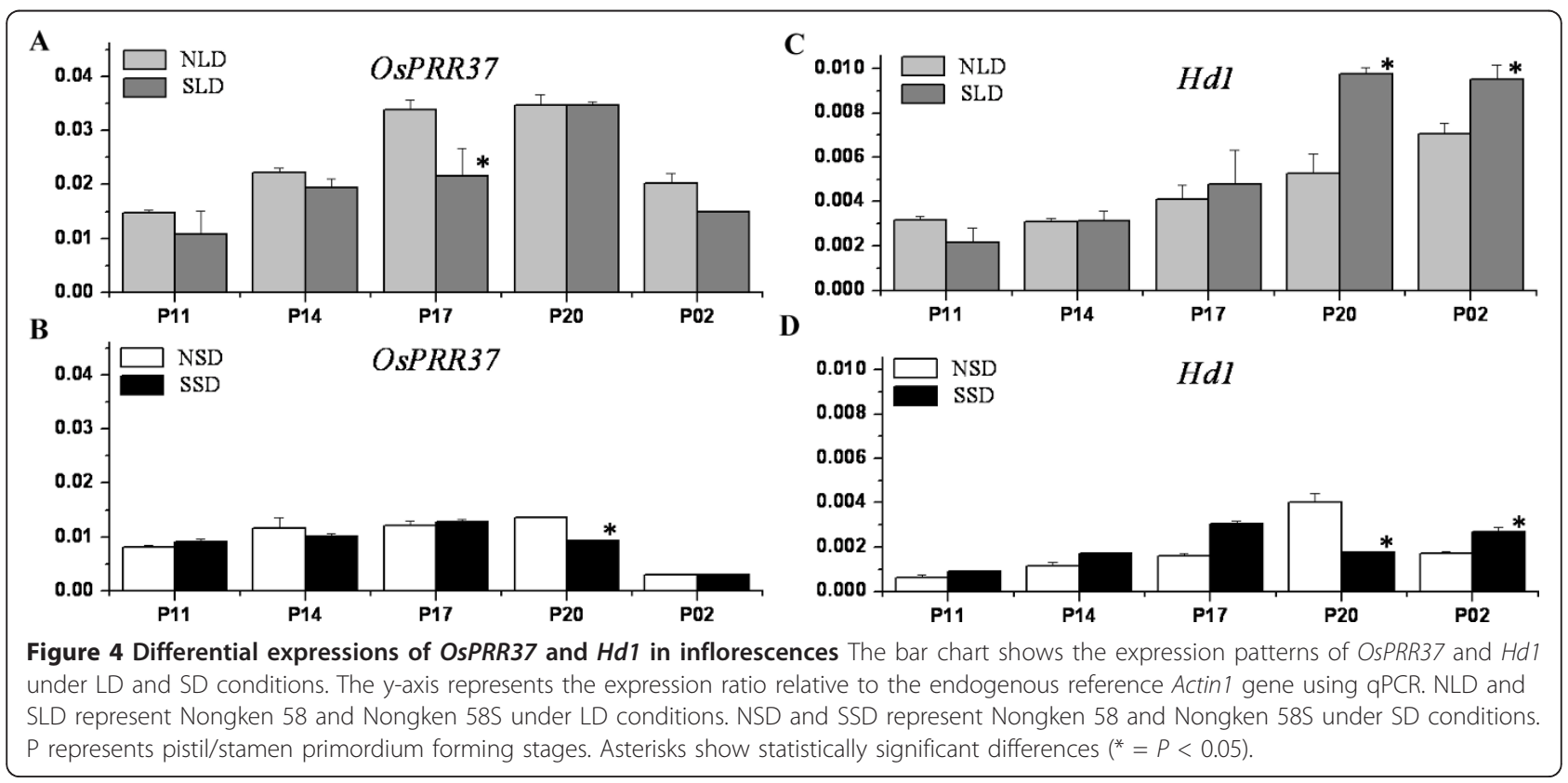


Moreover, the differences under SD conditions suggest a potential explanation for why pollen fertility cannot be completely recovered under SD in Nongken 58S.

\section{Discussion}

Circadian rhythm and light signals have been reported to be involved in several physiological processes, including flowering, various stress responses and metabolism $[26,30,31,38]$. However, this is the first report suggesting a role for circadian rhythm and flowering signals in the regulation network of male sterility. In general, these genes promote the transition from vegetative growth to reproductive growth under SD conditions in SD plants and under LD conditions in LD plants [23]. Although the male sterility transition in PGMS rice is known to be regulated by LD conditions, the mechanism of regulation remains unknown. Global gene expression profiling under LD conditions revealed a suppressive trend and categorised into the circadian rhythm and flowering pathways. Both pathways regulate the process in a coordinated manner. Further studies revealed that the diurnal expression profiles of OsPRR1, OsPRR37, OsGI, OsLHY, OsDof and Hd1 under LD conditions were reprogrammed.

In this study, OsPRR1, OsPRR37 and OsGI exhibited similar expression patterns in the leaf under LD conditions, suggesting that these three genes may function together. OsLHY and OsDof also exhibit similar differentially expressed patterns, suggesting that they may function coordinately. In Arabidopsis, TOC1/PRR1, LHY and GI, key component factors in the central clock, comprise interlocked transcriptional feedback loops to regulate plant circadian rhythms [12]. In PGMS rice, OsPRR1, $O s L H Y$ and $O s G I$ were not only involved in the circadian rhythm, but were also involved in the male sterility transition in coordination with OsPRR37 and OsDof. The LD signals maybe integrated and transmitted to the downstream genes through rice central clock in leaves. The day length signal may have been transmitted to the inflorescence via $H d 1$ and $O s P R R 37$, ultimately leading to pollen abortion.

In the rice flowering pathway, OsGI is upstream of $H d 1$ and is positively correlated with $H d 1$ expression. $H d 1$ is activated by OsGI under LD or SD conditions. $H d 1$ activates $H d 3 a / R F T 1$ expression under SD conditions to promote flowering. However, $H d 1$ suppresses Hd3a/RFT1 expression under LD conditions to inhibit flowering [24]. In PGMS rice, $H d 1$ expression may also have a positive correlation with OsGI under LD conditions in Nongken 58S, as its expression was activated after OsGI expression (Figure $3 \mathrm{C}, \mathrm{D}$ ). This implies that Hd1 may function downstream of OsGI in the male sterility transition in PGMS rice. In this case, $\mathrm{Hd} 3 \mathrm{a} /$ RFT1 may not be the downstream regulator of $H d 1$ since there were no rhythm differences between Nongken $58 \mathrm{~S}$ and Nongken 58 . Hd1 was activated by OsGI under LD conditions and suppressed the unknown factors. This may influence downstream gene expression and finally affect male fertility.

In leaves, the diurnal expression patterns of OsPRR1, OsPRR37, OsGI, OsLHY, OsDof and Hd1 showed significant differences between Nongken 58S and Nongken 58. In the inflorescence, only OsPRR37 and $H d 1$ exhibited different expression patterns between Nongken $58 \mathrm{~S}$ and Nongken 58 under both LD and SD. Therefore, it is speculated that the clock genes in leaves may function as sensors for day length. These genes may receive day length signals and integrate and transmit the signals into the inflorescence through a series of unknown pathways. Both OsPRR37 and Hd1 may be the effectors in the inflorescence, and they are likely involved in regulation of male sterility directly.

Previous studies have indicated that phytochromes and cryptochromes are involved in the male sterility transition. However, there were no diurnal expression differences in OsPhyA, OsPhyB, OsCryla, OsCry1b, OsCry 2 and OsCry3 under LD conditions between Nongken $58 \mathrm{~S}$ and Nongken 58 (data not shown). Using an ELISA assay, Wang, et al. found that phyA content in Nongken 58S leaves was higher than in Nongken 58 under the identical day length treatment [49]. The differences in phytochromes and cryptochromes may be at the protein level and needs further investigation.

\section{Conclusion}

In conclusion, the transcriptome of Nongken $58 \mathrm{~S}$ was significantly suppressed and the repression effects were markedly intensified when inflorescence development proceeded. Rice circadian rhythm genes OSPRR1, OsPRR37, OsGI, OsLHY and OsDof and the flowering gene $H d 1$ were coordinately involved in signal transduction in leaves. Furthermore, $H d 1$ and OsPRR37 may be signal sensors in inflorescences to directly affect the male sterility transition.

\section{Additional material}

Additional file 1: Table S1 Annotation of sample names. $G$ stands for glume primordium differentiation stage; $P$ stands for pistil/stamen primordium forming stage; S stands for Nongken 58S; SD is the abbreviation for short day condition and LD is the abbreviation for long day condition; 2 stands for 02:00 $\mathrm{h}$.

Additional file 2: Table S2 Primers used for qPCR. All of these primers were designed by Primer Express 3.0.

Additional file 3: Table S3 The Correlation coefficients between different biological repeats. The correlation coefficients are calculated by Prism 5.0 software. 1,2,3 represent three different biological repeats.

Additional file 4: Table S4 Differentially expressed genes in G-SSD2 vs. G-SLD2 data. All the DEGs with $F C \geq 2$ or $F C \leq 0.5$ are listed in this table. 
Additional file 5: Table S5 Differentially expressed genes in P-SSD2 vs. P-SLD2 data. All the DEGs with $F C \geq 2$ or $F C \leq 0.5$ are listed in this table.

Additional file 6: Figure S1 Diurnal expression profiles of OSPRR1, OsPRR37, OsGl, Hd1, OsLHY and OsDof genes in leaf. The diagram shows the diurnal expression profiles of OsPRR1, OsPRR37, OsGl, Hd1, OsLHY and OsDof genes under SD conditions. The $y$-axis represents the expression ratio relative to the endogenous reference Actin 1 gene using qPCR. The boxes on the $x$-axis represent day (blank) and night (solid). NSD and SSD represent Nongken 58 and Nongken 585 under SD conditions. $\mathrm{G}$ and $\mathrm{P}$ indicate the glume primordium differentiation and pistil/stamen primordium forming stages, respectively.

Additional file 7: Figure S2 Diurnal expression profiles of Ehd2, Ehd1, Hd3a, RFT1 and OsMADS1 under LD conditions in leaf. The diagram shows the circadian rhythm of the expression of Ehd2, Ehd1, Hd3a, RFT1 and OSMADS1 under LD conditions. The y-axis represents the expression ratio relative to the endogenous reference Actin 1 gene using qPCR. The boxes on the $x$-axis represent day (blank) and night (solid). NLD and SLD represent Nongken 58 and Nongken $58 S$ under LD conditions. $\mathrm{G}$ and $\mathrm{P}$ indicate the glume primordium differentiation and pistil/stamen primordium forming stages, respectively.

Additional file 8: Figure S3 Expression patterns of OsPRR1, OsGI, OsLHY and OsDof under LD and SD conditions in inflorescence. The bar chart shows the expression patterns of OsPRRI, OsGl, OsLHY and OsDof under LD and SD conditions in inflorescence. The $y$-axis represents the expression ratio relative to the endogenous reference Actin 1 gene using QPCR. NLD and SLD represent Nongken 58 and Nongken 585 under LD conditions. NSD and SSD represent Nongken 58 and Nongken $58 \mathrm{~S}$ under SD conditions. P indicates the pistil/stamen primordium forming stages. Asterisks show statistically significant differences $\left(^{*}=P<\right.$ $0.05)$.

\section{Acknowledgements and Funding}

This work was supported by the National Basic Research Program of China (973) 2011CB100102. We are grateful to Dr. Hexin Guan for technical assistance.

\section{Authors' contribution}

WW, ZL, ZG, GS, QC and DY conceived and designed the experiments; WW and $\mathrm{ZL}$ analysed the microarray data; WW, ZL, ZG, GS, DJ conducted portions of the experiments; WW, ZL, ZG, GS, YZ and DY prepared the manuscript. All authors have read and approved the final manuscript.

Received: 27 March 2011 Accepted: 25 September 2011

Published: 25 September 2011

\section{References}

1. Shi MS: The discovery and study of the photosensitive recessive malesterile rice. Scientia Agricultura Sinica 1985, 2:44-48.

2. Yuan SC, Zhang ZG, Xu CZ: Studies on the critical stage of fertility change induced by light and its phase development in HPGMR. Acta Agronomica Sinica 1988, 14:7-13.

3. Li XH, Wang FL, Lu Q, Xu CG: Fine Mapping of PSGMS Gene pms3 in Rice. Acta Agronomica Sinica 2002, 28:310-314

4. Mei MH, Chen L, Zhang Z, Li Z, Xu C, Zhang Q: pms3 is the locus causing the original photoperiod-sensitive male sterility mutation of 'Nongken 58S'. Science China Life Sciences 1999, 42:316-322.

5. Wang FP, Mei MH, Xu CG, Zhang QF: pms1 is not the Locus Relevant to Fertility Difference between the Photoperiod-sensitive Male Sterile Rice Nongken 58 and Normal Rice "Nongken 58". Acta Botanica Sinica 1997, 39:922-925.

6. Zhang Q, Shen BZ, Dai XK, Mei MH, Saghai Maroof MA, Li ZB: Using bulked extremes and recessive class to map genes for photoperiod-sensitive genic male sterility in rice. Proceedings of National Academic of Science 1994, 91:8675-8679.
7. Zhang XG, Zhu YG: A Genetic Study on Sterility of Hubei Photoperiod Sensitive Genic Male-Sterile Rice. Journal of Huazhong Agricultural University 1990, 9:481-483.

8. Liu N, Shan Y, Wang FP, Xu CG, Peng KM, Li XH, Zhang QF: Identification of an 85-kb DNA fragment containing pms1, a locus for photoperiodsensitive genic male sterility in rice. Molecular Genetics and Genomics 2001, 266:271-275

9. Lu Q, Li XH, Guo D, Xu CG, Zhang Q: Localization of pms3, a gene for photoperiod-sensitive genic male sterility, to a 28.4-kb DNA fragment. Molecular Genetics and Genomics 2005, 273:507-511.

10. Dunlap JC, Loros JJ, DeCoursey P: Chronobiology: Biological Timekeeping. 2004

11. McClung CR: Plant circadian rhythms. Plant Cell 2006, 18:792-803,

12. Harmer SL: The circadian system in higher plants. Annual Review of Plant Biology 2009, 60:357-377.

13. Somers DE, Webb AA, Pearson M, Kay SA: The short-period mutant, toc11 , alters circadian clock regulation of multiple outputs throughout development in Arabidopsis thaliana. Development 1998, 125:485-494.

14. Schaffer R, Ramsay N, Samach A, Corden S, Putterill J, Carre IA, Coupland G: The late elongated hypocotyl mutation of Arabidopsis disrupts circadian rhythms and the photoperiodic control of flowering. Cell 1998, 93:1219-1229.

15. Wang ZY, Tobin EM: Constitutive expression of the CIRCADIAN CLOCK ASSOCIATED 1 (CCA1) gene disrupts circadian rhythms and suppresses its own expression. Cell 1998, 93:1207-1217.

16. Locke JC, Southern MM, Kozma-Bognar L, Hibberd V, Brown PE, Turner MS, Millar AJ: Extension of a genetic network model by iterative experimentation and mathematical analysis. Molecular Systems Biology 2005, 1:2005 0013

17. Locke JC, Kozma-Bognar L, Gould PD, Feher B, Kevei E, Nagy F, Turner MS, Hall A, Millar AJ: Experimental validation of a predicted feedback loop in the multi-oscillator clock of Arabidopsis thaliana. Molecular Systems Biology 2006, 2:59

18. Komiya R, Yokoi S, Shimamoto K: A gene network for long-day flowering activates RFT1 encoding a mobile flowering signal in rice. Development 2009, 136:3443-3450

19. Komiya R, Ikegami A, Tamaki S, Yokoi S, Shimamoto K: Hd3a and RFT1 are essential for flowering in rice. Development 2008, 135:767-774.

20. Tamaki S, Matsuo S, Wong HL, Yokoi S, Shimamoto K: Hd3a protein is a mobile flowering signal in rice. Science 2007, 316:1033-1036.

21. Doi K, Izawa T, Fuse T, Yamanouchi U, Kubo T, Shimatani Z, Yano M, Yoshimura A: Ehd1, a B-type response regulator in rice, confers short-day promotion of flowering and controls FT-like gene expression independently of Hd1. Genes \& Development 2004, 18:926-936.

22. Kojima S, Takahashi Y, Kobayashi Y, Monna L, Sasaki T, Araki T, Yano M: $\mathrm{Hd} 3 \mathrm{a}$, a rice ortholog of the Arabidopsis FT gene, promotes transition to flowering downstream of $\mathrm{Hd} 1$ under short-day conditions. Plant \& Cell Physiology 2002, 43:1096-1105.

23. Izawa T: Daylength measurements by rice plants in photoperiodic shortday flowering. International Review of Cytology 2007, 256:191-222.

24. Hayama R, Yokoi S, Tamaki S, Yano M, Shimamoto K: Adaptation of photoperiodic control pathways produces short-day flowering in rice. Nature 2003, 422:719-722.

25. Yano M, Katayose $Y$, Ashikari M, Yamanouchi U, Monna L, Fuse T, Baba T, Yamamoto K, Umehara Y, Nagamura Y, Sasaki T: Hd1, a major photoperiod sensitivity quantitative trait locus in rice, is closely related to the Arabidopsis flowering time gene CONSTANS. Plant Cell 2000, 12:2473-2484

26. Turck F, Fornara F, Coupland G: Regulation and identity of florigen: FLOWERING LOCUS T moves center stage. Annual Review of Plant Biology 2008, 59:573-594.

27. Kobayashi Y, Weigel D: Move on up, it's time for change-mobile signals controlling photoperiod-dependent flowering. Genes \& Development 2007, 21:2371-2384.

28. Dodd AN, Salathia N, Hall A, Kevei E, Toth R, Nagy F, Hibberd JM, Millar AJ Webb AA: Plant circadian clocks increase photosynthesis, growth, survival, and competitive advantage. Science 2005, 309:630-633.

29. Yakir E, Hilman D, Harir Y, Green RM: Regulation of output from the plant circadian clock. FEBS Journal 2007, 274:335-345. 
30. McClung CR, Gutierrez RA: Network news: prime time for systems biology of the plant circadian clock. Current Opinion in Genetics \& Development 2010, 20:588-598.

31. de Montaigu A, Toth R, Coupland G: Plant development goes like clockwork. Trends in Genetics 2010, 26:296-306.

32. Doherty CJ, Kay SA: Circadian control of global gene expression patterns. Annual Review of Genetics 2010, 44:419-444.

33. Covington MF, Maloof JN, Straume M, Kay SA, Harmer SL: Global transcriptome analysis reveals circadian regulation of key pathways in plant growth and development. Genome Biology 2008, 9:R130.

34. Michael TP, Breton G, Hazen SP, Priest H, Mockler TC, Kay SA, Chory J: A morning-specific phytohormone gene expression program underlying rhythmic plant growth. PLoS Biology 2008, 6:e225.

35. Mizuno T, Yamashino T: Comparative transcriptome of diurnally oscillating genes and hormone-responsive genes in Arabidopsis thaliana: insight into circadian clock-controlled daily responses to common ambient stresses in plants. Plant \& Cell Physiology 2008, 49:481-487.

36. Nozue K, Covington MF, Duek PD, Lorrain S, Fankhauser C, Harmer SL, Maloof JN: Rhythmic growth explained by coincidence between internal and external cues. Nature 2007, 448:358-361.

37. Niwa $Y$, Yamashino T, Mizuno T: The circadian clock regulates the photoperiodic response of hypocotyl elongation through a coincidence mechanism in Arabidopsis thaliana. Plant \& Cell Physiology 2009, 50:838-854.

38. Roden LC, Ingle RA: Lights, rhythms, infection: the role of light and the circadian clock in determining the outcome of plant-pathogen interactions. Plant Cell 2009, 21:2546-2552.

39. Yang DC, Zhu YG: Primary study on total leaf RNA in PGMR at different photoperiod treatments and developmental stages. Acta Genetica Sinica 1990, 17:308-312.

40. Hulsegge I, Kommadath A, Smits MA: Globaltest and GOEAST: two different approaches for Gene Ontology analysis. BMC Proceeding 2009, 3(Suppl 4):S10.

41. Zheng Q, Wang XJ: GOEAST: a web-based software toolkit for Gene Ontology enrichment analysis. Nucleic Acids Research 2008, 36:W358-363.

42. Xue W, Xing Y, Weng X, Zhao Y, Tang W, Wang L, Zhou H, Yu S, Xu C, Li X, Zhang Q: Natural variation in Ghd7 is an important regulator of heading date and yield potential in rice. Nature Genetics 2008, 40:761-767.

43. Shi Y, Zhao S, Yao J: Premature tapetum degeneration: a major cause of abortive pollen development in photoperiod sensitive genic male sterility in rice. Journal of Integrative Plant Biology 2009, 51:774-781.

44. Xue GX, Zhao JZ: A Preliminary Study on the Critical-Daylength Evoking the Photoperiodic Sensitive Male Sterility of Rice and Their Responses to Other Environmental Factors. Acta Agronomica Sinica 1990, 16:112-122.

45. Hedegaard J, Arce C, Bicciato S, Bonnet A, Buitenhuis B, Collado-Romero M, Conley LN, Sancristobal M, Ferrari F, Garrido JJ, et al: Methods for interpreting lists of affected genes obtained in a DNA microarray experiment. BMC Proceeding 2009, 3(Suppl 4):S5.

46. Chen ZX, Wu JG, Ding WN, Chen HM, Wu P, Shi CH: Morphogenesis and molecular basis on naked seed rice, a novel homeotic mutation of OsMADS1 regulating transcript level of AP3 homologue in rice. Planta 2006, 223:882-890.

47. Agrawal GK, Abe K, Yamazaki M, Miyao A, Hirochika H: Conservation of the E-function for floral organ identity in rice revealed by the analysis of tissue culture-induced loss-of-function mutants of the OsMADS1 gene. Plant Mol Biol 2005, 59:125-135.

48. Prasad K, Parameswaran S, Vijayraghavan U: OsMADS1, a rice MADS-box factor, controls differentiation of specific cell types in the lemma and palea and is an early-acting regulator of inner floral organs. Plant J 2005, 43:915-928.

49. Wang W, Tong Z, Kuang TY, Tang PS: Immunoassay of Phytochrome A Content in Photoperiod-sensitive Genic Male-sterile Rice. Developmental and Reproductive Biology 1996, 5:51-59.

doi:10.1186/1471-2164-12-462

Cite this article as: Wang et al:: Comparative Transcriptomes Profiling of Photoperiod-sensitive Male Sterile Rice Nongken 58S During the Male Sterility Transition between Short-day and Long-day. BMC Genomics 2011 12:462.

\section{Submit your next manuscript to BioMed Central and take full advantage of:}

- Convenient online submission

- Thorough peer review

- No space constraints or color figure charges

- Immediate publication on acceptance

- Inclusion in PubMed, CAS, Scopus and Google Scholar

- Research which is freely available for redistribution

Submit your manuscript at www.biomedcentral.com/submit
C Biomed Central 